\title{
THE LIMITING AMPLITUDE PRINCIPLE FOR THE ACOUSTIC WAVE OPERATORS IN TWO UNBOUNDED MEDIA
}

By

\author{
Mitsuteru KADOWAKI
}

\section{Introduction.}

In our previous paper Kadowaki [3], we have proved the nonexistence of eigenvalues and the limiting absorption principle for the acoustic wave operators in two unbounded media. In the present paper we study the limiting amplitude principle for these operators. We assume that the propagation speed is discontinuous at the interface and the equilibrium density is 1 .

Let $n \geqq 3$ and $x=(y, z) \in \boldsymbol{R}^{n-1} \times \boldsymbol{R}$. We deal with the asymptotic behaviour (as $t \rightarrow+\infty$ ) of the solutions of the following Cauchy problem

$$
\left\{\begin{array}{l}
\partial_{t}^{2} u(t, x)-a(x)^{2} \Delta u(t, x)=\exp (-i t \sqrt{\omega}) f(x) \quad(t, x) \in \boldsymbol{R}_{+} \times \boldsymbol{R}^{n} \\
u(0, x)=\partial_{t} u(0, x)=0
\end{array}\right.
$$

where $\omega>0$.

We make the assumptions for the interface separating two media and $a(x)$.

Let $\varphi_{0}(y)=a|y|$ and $\varphi(y) \in C^{1}\left(\boldsymbol{R}^{n-1} \backslash 0\right)$, where $a \geqq 0$. We assume that $\varphi(y)$ describes the interface and satisfies

$$
\sum_{|\alpha| \leq 1}|y|^{|\alpha|}\left|\partial^{\alpha}\left(\varphi(y)-\varphi_{0}(y)\right)\right|=O\left(|y|^{-\theta}\right) \quad(|y| \rightarrow \infty),
$$

for some $\theta>0$, and

$$
\sum_{|\alpha| \leq 1}|y|^{|\alpha|}\left|\partial^{\alpha} \varphi(y)\right|=O\left(|y|^{-\sigma}\right) \quad(|y| \rightarrow 0),
$$

where $0<\sigma<1 / 2$. For $\varphi(y)$, we use the following notation

$$
\begin{gathered}
\Omega_{+}=\{x=(y, z): z>\varphi(y)\}, \\
\Omega_{-}=\{x=(y, z): z<\varphi(y)\}, \\
S=\{x=(y, z): z=\varphi(y)\} .
\end{gathered}
$$

We denote the unit normal vector at the point $x \in S$ by $\nu=\left(\nu_{1}, \nu_{2}, \cdots, \nu_{n-1}, \nu_{z}\right)$

Received September 9, 1992. Revised March 16, 1993. 
with $\nu_{z}>0$.

The propagation speed $a(x)>0$ is assumed to satisfy following

$$
1 / c<a(x)<c
$$

for some $c>1$, and there exist $a_{ \pm}>0, a_{L}^{ \pm}(x) \in \boldsymbol{B}^{1}\left(\Omega_{ \pm}\right)$and $a_{S}(x) \in L^{\infty}\left(\boldsymbol{R}^{n}\right)$ such that $a(x)$ is decomposed as

$$
\left\{\begin{array}{l}
a(x)=a_{ \pm}+a_{L}^{ \pm}(x)+a_{S}(x) \quad\left(x \in \Omega_{ \pm}\right), \\
\sum_{|\alpha| \leq 1}|x|^{|\alpha|}\left|\partial^{\alpha} a_{L}^{ \pm}(x)\right|=O\left(|x|^{-\theta}\right) \quad\left(|x| \rightarrow+\infty, x \in \Omega_{ \pm}\right), \\
a_{S}(x)=O\left(|x|^{-\theta-1}\right) \quad(|x| \rightarrow+\infty),
\end{array}\right.
$$

for some $\theta>0$.

Under (A.0) (A.3), we show the limiting amplitude principle of the acoustic operator, $-a(x)^{2} \Delta$, for (1.1).

There are many works dealing with the limiting amplitude principle for the acoustic operators. For example Eidus [2] has proved the limiting absorption and amplitute principle for two unbounded media problem with the interface satisfying the following condition: for any $x \in S$

$$
\begin{aligned}
& \nu_{2} \geqq C_{1}>0 \\
& |x \cdot \nu| \leqq C_{2}
\end{aligned}
$$

where $C_{j}>0(j=1,2)$, are independent of $x \in S$. For example,

$$
\varphi(y) \in C^{1}\left(\boldsymbol{R}^{n-1}\right), \varphi(y)=\frac{\sin |y|}{|y|}(y \gg 1), \varphi_{0}(y)=0
$$

satiafies (1.2) and (1.3), but not satisfies (A.0). We can also deal with the following interface not satisfying (1.2),

$$
\varphi(y)=|y|^{-\sigma}, \quad \varphi_{0}(y)=0
$$

where, $0<\sigma<1 / 2$. The propagation speed considered in Eidus [2] is a piecewise constant function while we can perturb the propagation speed. Tamura [7] has proved the limiting amplitude principle for the acoustic wave operators in inhomogeneous media. Kikuchi and Tamura [4] have also proved this principle for the acoustic wave operators in perturbed stratified fluilds.

In order to show the limiting amplitude principle for our operator, we need a low frequency behaviour of the resolvent (see Eidus [1]). In [4] and [7], they use Mourre's method to show a low frequency behaviour of the resolvent and show the limiting amplitude principle. We also use Mourre's method to show a low frequency behaviour of the resolvent of our operator (Theorem 1.1). 
We now define the acoustic operator $L$ as

$$
L=-a(x)^{2} \Delta
$$

Under the above assumption, (A.0) (A.3), $L$ is symmetric operator in the Hibert space $L^{2}\left(\boldsymbol{R}^{n} ; a(x)^{-2} d x\right)$ and admits a unique self-adjoint realization. We denote by the same notation $L$ this self-adjoint realization. Then $L$ is a positive operator (zero is not an eigenvalue) and the domain $D(L)$ is given by $D(L)=$ $H^{2}\left(\boldsymbol{R}^{n}\right), H^{s}\left(\boldsymbol{R}^{n}\right)$ being the Sobolev space of order $s$ over $\boldsymbol{R}^{n}$. We also denote by $R(z ; L)$ the resolvent $(L-z)^{-1}$ of $L$ for $\operatorname{Im} z \neq 0$.

We need several notations to describe our results. Let $L^{2}$ be the usual $L^{2}$ space defined on $\boldsymbol{R}^{n}$, with the inner product

$$
\langle u, v\rangle=\int u(x) \overline{v(x)} d x
$$

and the corresponding norm $|\cdot|_{0}$. For $\alpha \in \boldsymbol{R}$, let $L_{\alpha}^{2}$ be the weighted $L^{2}$ space defined by

$$
L_{\alpha}^{2}=\left\{u(x):\langle x\rangle^{\alpha} u(x) \in L^{2}\left(\boldsymbol{R}^{n}\right)\right\},\langle x\rangle=\left(1+|x|^{2}\right)^{1 / 2},
$$

with the norm

$$
|u|_{\alpha}^{2}=\int\langle x\rangle^{2 \alpha}|u(x)|^{2} d x .
$$

Let $A: L_{\alpha}^{2} \rightarrow L_{\beta}^{2}$ be a bounded operator. We denote by $\|A\|_{\alpha \rightarrow \beta}$ the operator norm considered as an operator from $L_{\alpha}^{2}$ to $L_{\beta}^{2}$. If, in particular, $A: L^{2} \rightarrow L^{2}$ is considered as an operator from $L^{2}$ into itself, then its norm is denoted by the simplified notation $\|A\|$.

In Kadowaki [3], we have obtained the following result

ThEOREm 1.0. Assume that (A.0) (A.3). Then

(i) L has no eigenvalues.

(ii) Let $\lambda_{0}>0$ and $\alpha>1 / 2$. Then there exists some compact interval $I \subset \boldsymbol{R}_{+}$ containing $\lambda_{0}$ and $a$ positive constant $C=C\left(\lambda_{0}, \alpha\right)$ such that

$$
\left\|\langle x\rangle^{-\alpha} R(\lambda \pm i \kappa ; L)\langle x\rangle^{-\alpha}\right\| \leqq C,
$$

for $\lambda \in I, 0<\kappa<1$.

(iii) For every $\lambda>0$ and $\alpha>1 / 2$, the following two limits

$$
R(\lambda \pm i 0 ; L)=\lim _{\kappa \downarrow 0} R(\lambda \pm i \kappa ; L),
$$

exist in the uniform operator topology of $\boldsymbol{B}\left(L_{a}^{2}, L_{-a}^{2}\right)$. Moreover $R(\lambda \pm i 0 ; L)$ are locally Hölder continuous. 
The aim here is to prove the following.

THEOREM 1.1. Assume that (A.0) (A.3), Let $\alpha>1$ and $\beta>1 / 2$. Then there exists $d, 0<d<1 / 2$, such that

$$
\|R(\lambda \pm i 0 ; L)\|_{\beta \rightarrow-a}=O\left(\lambda^{-d}\right), \quad(\lambda \rightarrow 0) .
$$

By Theorem 1.0 and 1.1, we have the following theorem (see Eidus [1] or Tamura [7]).

ThEOREM 1.2 (LIMITING AMPLITUde PRINCIPLE). Assume that (A.0) (A.3). Let $u=u(t, x)$ be the solution of (1.1) with $f \in L_{\beta}^{2}, \beta>1 / 2$. Then $u(t, x)$ behaves like

$$
u=\exp (-i t \sqrt{\omega}) R(\omega+i 0 ; L) f+o(1), \quad(t \rightarrow \infty)
$$

strongly in $L_{-\alpha}^{2}, \alpha>1$.

Acknowledgement. The author would like to express his sincere gratitute to Professors M. Matsumura and K. Kajitani for their generous advice and kind encouragement.

\section{Reduction to main lemma.}

We consider only the case $1=a_{-}^{-2}<a_{+}^{-2}$. The other cases can be proved similarly. We define the self-adjoint operator $H(\lambda)$ on $L^{2}$ by

Then we have

$$
\left\{\begin{array}{l}
H(\lambda)=-\Delta-\lambda\left(a^{-2}(x)-1\right) \\
D(H(\lambda))=H^{2}\left(\boldsymbol{R}^{n}\right)
\end{array}\right.
$$

$$
R(\lambda \pm i \kappa ; L)=Q(\lambda, \pm i \kappa ; H(\lambda)) a^{-2}(x),
$$

where $Q(\lambda, \pm i \kappa ; H(\lambda))=\left(H(\lambda)-\lambda \mp i \kappa a^{-2}(x)\right)^{-1}$. Therefore, Theorem 1.1 is obtained as an immediate consequence of the following.

LEMMA 2.1. Let the pair $(\alpha, \beta)$ be as in Theorem 1.1. Then

$$
\|Q(\lambda, \pm i 0 ; H(\lambda))\|_{\beta \rightarrow-\alpha}=O\left(\lambda^{-d}\right), \quad(\lambda \rightarrow 0),
$$

for some $d, 0<d<1 / 2$.

By the assumption (A.3), we can decompose $a^{-2}(x)$ as $a^{-2}(x)=E_{1}^{ \pm}(x)+$ $E_{2}(x)\left(x \in \Omega_{ \pm}\right)$in such a way:

$$
\sum_{|\alpha| \leq 1}|x|^{|\alpha|}\left|\partial_{x}^{\alpha}\left(E_{1}^{ \pm}(x)-a_{ \pm}^{-2}\right)\right|=O\left(|x|^{-\theta}\right) \quad\left(|x| \rightarrow \infty, x \in \Omega_{ \pm}\right),
$$




$$
\begin{gathered}
E_{2}(x)=O\left(|x|^{-1-\theta}\right) \quad(|x| \rightarrow \infty), \\
\sum_{|\alpha| \leqq 1}\langle x\rangle^{|\alpha|}\left|\partial_{x}^{\alpha}\left(E_{1}^{ \pm}(x)-a_{ \pm}^{-2}\right)\right| \leqq \delta_{0} \quad\left(x \in \Omega_{ \pm}\right),
\end{gathered}
$$

for $\delta_{0}>0$ small enough, $\delta_{0}$ being fixed throughout.

LEMMA 2.2. Let $H_{1}(\lambda)=-\Delta-\lambda\left(E_{1}(x)-1\right)$, and $\alpha>1$. Then, we have

$$
\left\|\left(H_{1}(\lambda)-\lambda \mp i \kappa a^{-2}(x)\right)^{-1}\right\|_{\alpha \rightarrow-\alpha}=O(1), \quad(\lambda \rightarrow 0),
$$

uniformly in $\kappa>0$ small enough, where $E_{1}(x)=E_{1}^{ \pm}(x)\left(x \in \Omega_{ \pm}\right)$

We show that Lemma 2.1 follows from Lemma 2.2. We need the following two lemmas to estimate an integral on the interface $S$.

LEMMA 2.3. Let $s>1 / 2$. For $u \in \boldsymbol{S}\left(\boldsymbol{R}^{n}\right)$ (Schwartz space), we define

$$
\left(T_{\varphi} u\right)(y)=u(y, \varphi(y))
$$

$T_{\varphi}$ has an extension to a bounded operator from $H^{s}\left(\boldsymbol{R}^{n}\right)$ to $L^{2}\left(\boldsymbol{R}^{n-1}\right)$.

For a proof of Lemma 2.3, see that of Lemma 2.1 of Kadowaki [3].

LEMMA 2.4 (SOBOLEv's THOREOM). Suppose that

$$
1 / 2-l / m=1 / q, \quad 2<q<\infty .
$$

Then we have the embedding

$$
H^{l}\left(\boldsymbol{R}^{m}\right) \subset L^{q}\left(\boldsymbol{R}^{m}\right) .
$$

Proof of Lemma 2.1. We prove Lemma 2.1 in the same way as in the proof of Lemma 1.1 of Tamura [7]. We assume (2.4), Let $\alpha>1$ and $\beta>1 / 2$. We assert that

$$
\begin{aligned}
& \left\|\left(H_{1}(\lambda)-\lambda \mp i \kappa a^{-2}(x)\right)^{-1}\right\|_{\beta \rightarrow-\alpha}=O\left(\lambda^{-1 / 2}\right), \quad(\lambda \rightarrow 0), \\
& \left\|\left(H_{1}(\lambda)-\lambda \mp i \kappa a^{-2}(x)\right)^{-1}\right\|_{\beta \rightarrow-\beta}=O\left(\lambda^{-1}\right), \quad(\lambda \rightarrow 0),
\end{aligned}
$$

uniformly in $\kappa>0$ small enough. By (2.5), (2.6) and Theorem 1.0(iii), we have Lemma 2.1 (see the proof of Lemma 1.1 of Tamura [7]). We prove the assertions (2.5) and (2.6). We consider the + case only. Let $u=\left(H_{1}(\lambda)-\lambda+i \kappa a^{-2}(x)\right)^{-1} f$ with $f \in L^{2}$. Then $u$ satisfies

$$
\left(-\Delta-\lambda E_{1}(x)+i \kappa a^{-2}(x)\right) u=f .
$$

We take the $L^{2}$ scalar product of $\langle x\rangle^{-\gamma} u$ with equation (2.7), where $\gamma \geqq 0$. Integrating by part and taking real part, we have 


$$
\begin{aligned}
& \left\langle\langle x\rangle^{-r} \nabla u, \nabla u\right\rangle-1 / 2\left\langle u,\left(\nabla\langle x\rangle^{-r}\right) u\right\rangle \\
& =\lambda\left\langle E_{1} u,\langle x\rangle^{-r} u\right\rangle+R e\left\langle f,\langle x\rangle^{-r} u\right\rangle .
\end{aligned}
$$

Let $\delta<\min \{\theta, \sigma\}, 0<\delta \ll 1$, be fixed arbitrarily. Let $\chi(x)=\left\{\chi^{j}\right\}_{1 \leq j \leq n}$ be a real smooth vector field such that

$$
\chi^{j}(x)= \begin{cases}\left(1-|x|^{-\delta}\right) y_{j} /|x| & (j=1,2,3, \cdots, n-1) \\ \left(1-|x|^{-\delta}\right) z /|x| & (j=n)\end{cases}
$$

for $|x|>R$ and

$$
\operatorname{supp} \chi^{j} \subset\left\{|x|>R^{\prime}\right\},
$$

where $R>R^{\prime} \gg 1$. We use the summation convention. Noting that

$$
\begin{aligned}
& \sum_{j=1}^{n} \partial_{j} \chi^{j}\left|\partial_{j} u\right|^{2}=\left(\frac{1}{|x|}-\frac{1}{|x|^{\delta+1}}\right)|\nabla u|^{2} \\
& +\frac{\delta+1}{|x|^{\delta+3}} \sum_{j=1}^{n} x_{j}^{2}\left|\partial_{j} u\right|^{2}-\frac{1}{|x|^{3}} \sum_{j=1}^{n} x_{j}^{2}\left|\partial_{j} u\right|^{2} \quad(|x|>R)
\end{aligned}
$$

and

$$
\begin{aligned}
& \left|\sum_{j, k=1, j \neq k}^{n} \operatorname{Re}_{k} \chi^{j} \partial_{k} u \overline{\partial_{j} u}\right| \\
& \leqq\left(\frac{1}{|x|^{3}}-\frac{\delta+1}{|x|^{\delta+3}}\right)_{j, k=1, j \neq k} x_{j}^{n}\left|\partial_{k} u\right|^{2} \quad(|x|>R),
\end{aligned}
$$

we have

$$
R e \partial_{k} \chi^{j} \partial_{k} u \overline{\partial_{j} u} \geqq C_{\delta}\langle x\rangle^{-1-\delta}|\nabla u|^{2}, \quad|x|>R .
$$

We take the $L^{2}$ scalar product of $\chi \cdot \nabla u+(1 / 2)(\nabla \cdot \chi) u$ with equation (2.7). we have

$$
\begin{aligned}
\langle-\Delta u, & \chi \cdot \nabla u+(1 / 2)(\nabla \cdot \chi) u\rangle \\
& -\lambda\left\langle E_{1} u, \chi \cdot \nabla u+(1 / 2)(\nabla \cdot \chi) u\right\rangle \\
& +i \kappa\left\langle a^{-2}(\cdot) u, \chi \cdot \nabla u+(1 / 2)(\nabla \cdot \chi) u\right\rangle \\
= & \langle f, \chi \cdot \nabla u+(1 / 2)(\nabla \cdot \chi) u\rangle .
\end{aligned}
$$

First, we calculate the first term of left side of (2.10). Integrating by parts, we have

$$
\begin{aligned}
R e & \langle-\Delta u, \chi \cdot \nabla u+(1 / 2)(\nabla \cdot \chi) u\rangle \\
& =\operatorname{Re}\left\langle\left(\partial_{k} \chi^{j}\right) \partial_{k} u, \partial_{j} u\right\rangle-1 / 4\left\langle\left(\partial_{k}^{2} \partial_{j} \chi^{j}\right) u, u\right\rangle .
\end{aligned}
$$

In orded to calculate the second term of left side of (2.10), we set $w_{n}=z-\varphi(y)$ and $w^{\prime}=\left(y_{1}, y_{2}, y_{3}, \cdots, y_{n-1}\right)$. Then we have 


$$
\frac{D(y, z)}{D\left(w^{\prime}, w_{n}\right)}=\left|\begin{array}{ccccc}
1 & 0 & \cdots & 0 & 0 \\
0 & 1 & \cdots & 0 & 0 \\
\vdots & \vdots & \ddots & \vdots & \vdots \\
0 & 0 & \cdots & 1 & 0 \\
-\partial_{1} \varphi\left(w^{\prime}\right) & -\partial_{2} \varphi\left(w^{\prime}\right) & \cdots & -\partial_{n-1} \varphi\left(w^{\prime}\right) & 1
\end{array}\right|=1 .
$$

Thus, integrating by parts, we have (for detail, see the proof of Lemma 2.2 of Kadowaki [3])

$$
\begin{aligned}
& R e\left\langle E_{1} u, \chi \cdot \nabla u+(1 / 2)(\nabla \cdot \chi) u\right\rangle=-1 / 2\langle F u, u\rangle \\
& \quad+1 / 2 \int_{R^{n-1}}\left(\chi^{y}(y, \varphi(y)) \cdot \nabla_{y} \varphi(y)-\chi^{n}(y, \varphi(y))\right)\left(E_{1}^{+0}-E_{1}^{-0}\right)\left|T_{\varphi} u\right|^{2} d y,
\end{aligned}
$$

where $\chi^{y}=\left\{\chi^{j}\right\}_{1 \leqq j \leqq n-1}, F=\chi \cdot \nabla E_{1}(x)\left(x \in \Omega_{ \pm}\right)$and $E_{1}^{ \pm 0}=E_{1}(y, \varphi(y) \pm 0)$.

Noting that (2.11) and (2.12), we take the real part of (2.10). Then we have

$$
\begin{aligned}
& \left.R e\left\langle\partial_{k} \chi^{j}\right) \partial_{k} u, \partial_{j} u\right\rangle-1 / 4\left\langle\left(\partial_{k}^{2} \partial_{j} \chi^{j}\right) u, u\right\rangle+\lambda / 2\langle F u, u\rangle \\
& \quad-\lambda / 2 \int_{R^{n-1}}\left(\chi^{y}(y, \varphi(y)) \cdot \nabla_{y} \varphi-\chi^{n}(y, \varphi(y))\right)\left(E_{1}^{+0}-E_{1}^{-0}\right)\left|T_{\varphi} u(y)\right|^{2} d y \\
& =\operatorname{Re}\langle f, \chi \cdot \nabla u+(1 / 2)(\nabla \cdot \chi) u\rangle+\kappa \operatorname{Im}\left\langle a^{-2}(\cdot) u, \chi \cdot \nabla u\right\rangle,
\end{aligned}
$$

We set $\alpha=1+\delta$ and $\beta=(1+\delta) / 2$. Assume that $f \in L_{\alpha}^{2}$. In order to prove (2.5), we first estimate the fourth term of the left side of (2.13). Take $0<r_{0} \ll 1$ and $R_{0} \gg 1$ such that if $|y|<r_{0}$ or $|y|>R_{0}$, we have $|y|^{2}+|\varphi(y)|^{2}>R$. Using $r_{0}$ and $R_{0}$, we decompose the fourth term as

$$
\begin{aligned}
\int_{R^{n-1}} & \left(\chi^{y}(y, \varphi(y)) \cdot \nabla_{y} \varphi-\chi^{n}(y, \varphi(y))\right)\left(E_{1}^{+0}-E_{1}^{-0}\right)\left|T_{\varphi} u\right|^{2} d y \\
= & \int_{|y|<r_{0}} \frac{1-\left(|y|^{2}+|\varphi(y)|^{2}\right)^{-\delta / 2}}{\left(|y|^{2}+|\varphi(y)|^{2}\right)^{1 / 2}}\left(y \cdot \nabla_{y} \varphi-\varphi\right)\left(E_{1}^{+0}-E_{1}^{-0}\right)\left|T_{\varphi} u\right|^{2} d y \\
& +\int_{r_{0}<|y|<R_{0}}\left(\chi^{y}(y, \varphi(y)) \cdot \nabla_{y} \varphi(y)-\chi^{n}(y, \varphi(y))\right)\left(E_{1}^{+0}-E_{1}^{-0}\right)\left|T_{\varphi} u\right|^{2} d y \\
& +\int_{|y|>R_{0}} \frac{1-\left(|y|^{2}+|\varphi(y)|^{2}\right)^{-\delta / 2}}{\left(|y|^{2}+|\varphi(y)|^{2}\right)^{1 / 2}}\left(y \cdot \nabla_{y} \varphi-\varphi\right)\left(E_{1}^{+0}-E_{1}^{-0}\right)\left|T_{\varphi} u\right|^{2} d y \\
= & I_{1}+I_{2}+I_{3} .
\end{aligned}
$$

If $|y|<r_{0}$, there exist some $C_{1}>0$ such that $|\varphi(y)|>C_{1}>0$. Then we have

$$
\begin{aligned}
& I_{1} \leqq C \int_{|y|<r_{0}} \frac{\mid 1-\left(|y|^{2}+|\varphi(y)|^{2}\right)^{-\delta / 2}}{\left(|y|^{2}+|\varphi(y)|^{2}\right)^{1 / 2}}\left|y \cdot \nabla_{y} \varphi-\varphi\right|\left|T_{\varphi} u\right|^{2} d y \\
& \quad \leqq C \int_{|y|<r_{0}}|y|^{-\sigma(1+\sigma)}\left|T_{\varphi} v\right|^{2} d y
\end{aligned}
$$

where $v=\langle x\rangle^{-(1+\sigma) / 2} u$. 
If $0<\sigma<1 / 2$, there exist some $s>1 / 2$ and $p>n-1$ such that $n-1-\sigma(1+\sigma) p$ $>0$ and $(n-1) / 2 p+s=1$. Thus, by Lemma 2.4 and Hölder inequlity, we have

$$
\begin{aligned}
\int_{|y|<r_{0}}|y|^{-\sigma(1+\sigma)}\left|T_{\varphi} v\right|^{2} d y \\
\quad \leqq C \int_{-\infty}^{+\infty}\left(1+\zeta^{2}\right)^{s} \int_{|y|<r_{0}}|y|^{-\sigma(1+\sigma)}\left|\left(\mho_{z} v\right)(y, \zeta)\right|^{2} d y d \zeta \\
\quad \leqq C r_{0}^{(n-1-\sigma(1+\sigma)) / 2 p} \int_{-\infty}^{+\infty}\left(1+\zeta^{2}\right)^{s}\left|\left(\mathfrak{\mho}_{z} v\right)(\cdot, \zeta)\right|_{L^{2} p /(p-1)\left(R^{n-1)}\right.}^{2} d \zeta \\
\quad \leqq C r_{0}^{(n-1-\sigma(1+\sigma)) / 2 p} \int_{-\infty}^{+\infty}\left(1+\zeta^{2}\right)^{8}\left|\left(\mathfrak{\mho}_{z} v\right)(\cdot, \zeta)\right|_{H}^{2}(n-1) / 2 p\left(R^{n-1)}\right) d \zeta \\
\leqq C r_{0}^{(n-1-\sigma(1+\sigma)) / 2 p}\left(|u|^{2}(1+\sigma) / 2+|\nabla u|_{-(1+\sigma) / 2}^{2}\right),
\end{aligned}
$$

where $\mathfrak{F}_{z} v(\zeta)=(2 \pi)^{-1 / 2} \int_{-\infty}^{+\infty} e^{-i \zeta \cdot z} v(z) d z$. By Lemma 2.3 and (A.0), we also have the following

$$
\begin{aligned}
& I_{2} \leqq C \int_{r_{0<|y|<R_{0}}}\left|T_{\varphi} u\right|^{2} d y \leqq C\left(|u|_{-(1+\sigma) / 2}^{2}+|\nabla u|_{-(1+\sigma) / 2}^{2}\right), \\
& I_{3} \leqq C \int_{|y|>R_{0}} \frac{\left|y \cdot \nabla_{y} \varphi-\varphi\right|}{\left(|y|^{2}+|\varphi(y)|^{2}\right)^{1 / 2}}\left|T_{\varphi} u\right|^{2} d y \leqq C\left(|u|_{-(1+\theta) / 2}^{2}+|\nabla u|_{-(1+\theta) / 2}^{2} .\right.
\end{aligned}
$$

Thus, we have

$$
\begin{aligned}
& \left.\left|\int_{R^{n-1}}\left(\chi^{y}(y, \varphi(y)) \cdot \nabla_{y} \varphi(y)-\chi^{n}(y, \varphi(y))\right)\left(E_{1}^{+0}-E_{1}^{-0}\right)\right| T_{\varphi} u\right|^{2} d y \mid \\
& \leqq C\left(|u|^{2}(1+\theta) / 2+|\nabla u|_{-(1+\theta) / 2}^{2}+|u|_{-(1+\sigma) / 2}^{2}+|\nabla u|_{-(1+\sigma) / 2}^{2}\right)
\end{aligned}
$$

Following Lemma 1.1 of Tamura [7], we estimate the other terms of (2.13). By (2.9), we have

$$
R e \int_{|x|>R}\left(\partial_{k} \chi^{j}\right) \partial_{k} u \bar{u} \bar{u} d x \geqq C_{\delta} \int_{|x|>R}\langle x\rangle^{-1-\delta}|\nabla u|^{2} d x .
$$

By Lemma 2.2, we have

$$
\left|\left\langle\left(\partial_{k}^{2} \partial_{f} \chi^{j}\right) u, u\right\rangle\right| \leqq C|f|_{\alpha}^{2}
$$

By (2.1), we also have

$$
|\langle F u, u\rangle| \leqq C|u|^{2}(1+\theta) / 2 .
$$

Noting Lemma 2.2, we use (2.8) with $\gamma=2 \alpha$ to obtain

$$
|\nabla u|_{-\alpha} \leqq C|f|_{\alpha} .
$$

It follows from (2.18) and Lemma 2.2 that

$$
|R e\langle f, \chi \cdot \nabla u+(1 / 2)(\nabla \cdot \chi) u\rangle| \leqq C|f|_{\alpha}^{2} .
$$


We again use (2.8) with $\gamma=0$ to obtain

$$
\begin{gathered}
|\nabla u|_{0}^{2}=\lambda\left\langle E_{1} u, u\right\rangle+R e\langle f, u\rangle, \\
\kappa\left\langle a^{-2}(\cdot) u, u\right\rangle=\operatorname{Im}\langle f, u\rangle .
\end{gathered}
$$

By (A.2), (2.21) and Lemma 2.2, we have

$$
\kappa|u|_{0}^{2} \leqq C|f|_{\alpha}^{2} .
$$

Thus (2.20), (2.22) and Lemma 2.2 imply

$$
\left|\kappa \operatorname{Im}\left\langle a^{-2}(\cdot) u, \chi \cdot \nabla u\right\rangle\right| \leqq C|f|_{\alpha}^{2} .
$$

Since $\beta<(1+\theta) / 2,(1+\sigma) / 2$, we have by $(2.13) \sim(2.17),(2.19)$ and $(2.23)$

$$
|\nabla u|_{-\beta}^{2} \leqq C \lambda\left(|u|_{-(1+\theta) / 2}^{2}+|u|_{-(1+\sigma) / 2}^{2}\right)+C|f|_{\alpha}^{2} .
$$

Using (2.24) and (2.8) with $\gamma=2 \beta$, we have

$$
\lambda|u|_{-\beta}^{2} \leqq C \lambda\left(|u|_{-(1+\theta) / 2}^{2}+|u|_{-(1+\sigma) / 2}^{2}\right)+C|f|_{\alpha}^{2} .
$$

Again since $\beta<(1+\theta) / 2,(1+\sigma) / 2$, (2.25) implies

$$
\lambda|u|_{-\beta}^{2} \leqq C|f|_{\alpha}^{2}
$$

Considering the adjoint operator, we have (2.5). To prove (2.6), we repeat the same argument as above. Assume that $f \in L_{\beta}^{2}$. Then, by the same way as in the proof of (2.24), we can show that

$$
\begin{aligned}
|\nabla u|_{-\beta}^{2} \leqq & C \lambda\left(|u|_{-(1+\theta) / 2}^{2}+|u|_{\underline{2}_{(1+\sigma) / 2}}\right) \\
& +C\left(|u|_{-\alpha}^{2}+|f|_{\beta} \cdot|\nabla u|_{-\beta}+|f|_{\beta} \cdot|u|_{-\beta}\right) .
\end{aligned}
$$

Using (2.5) and (2.8) with $\gamma=2 \beta$, we have

$$
\lambda|u|_{-\beta}^{2} \leqq C\left(|\nabla u|_{-\beta}^{2}+\lambda^{-1}|f|_{\beta}^{2}\right) .
$$

Noting that $\beta<(1+\theta) / 2,(1+\sigma) / 2$, we have by $(2.26)$ and $(2.27)$

$$
|u|_{-\beta} \leqq C \lambda^{-1}|f|_{\beta} .
$$

The proof of Lemma 2.1 is now complete.

\section{Proof of Lemma 2.2.}

In this section we prove Lemma 2.2 by making use of commutator method developed by Mourre [5]. Let $A$ be the generator of the dilation unitary group:

$$
A=\frac{1}{2 i}(x \cdot \nabla+\nabla \cdot x) \text {. }
$$

We define the commutator $i\left[H_{1}(\lambda), A\right]$ as a form on $H^{2}\left(\boldsymbol{R}^{n}\right) \cap D(A)$ as follows: 
For $u, v \in H^{2}\left(\boldsymbol{R}^{n}\right) \cap D(A)$,

$$
\left\langle i\left[H_{1}(\lambda), A\right] u, v\right\rangle=i\left(\left\langle A u, H_{1}(\lambda) v\right\rangle-\left\langle H_{1}(\lambda) u, A v\right\rangle\right) .
$$

Lemma 3.1. The form $i\left[H_{1}(\lambda), A\right]$ defined on $H^{2}\left(\boldsymbol{R}^{n}\right) \cap D(A)$ is extended to a bounded operator from $H^{1}\left(\boldsymbol{R}^{n}\right)$ to $H^{-1}\left(\boldsymbol{R}^{n}\right)$ which is denoted by $i\left[H_{1}(\lambda), A\right]^{0}$. Moreover we have

$$
i\left[H_{1}(\lambda), A\right]^{0}=-2 \Delta+\lambda\left(F_{1}-T_{\varphi}^{*}\left(E_{1}^{+0}-E_{1}^{-0}\right)\left(y \cdot \nabla_{y} \varphi(y)-\varphi(y)\right) T_{\varphi}\right),
$$

where $F_{1}=x \cdot \nabla E_{1}(x)\left(x \in \Omega_{ \pm}\right)$.

For a proof of Lemma 3.1, see that of Lemma 2.2 of Kadowaki [3].

If $|\mu|>1,(A+i \mu)^{-1}$ sends $H^{k}\left(\boldsymbol{R}^{n}\right)$ into $H^{k}\left(\boldsymbol{R}^{n}\right)$ and as an operator on $H^{k}\left(\boldsymbol{R}^{n}\right)(k=0, \pm 1, \pm 2)$

$$
s-\lim _{|\mu| \rightarrow+\infty} i \mu(A+i \mu)^{-1}=I
$$

is valid (see Lemma 2.3 of Weder [9]). Put $A(\mu)=i \mu A(A+i \mu)^{-1}$. Using (3.1), we can prove the following lemma in the same way as the proof of Lemma 2.4 of Weder [9].

LEMMA 3.2 .

and

$$
i\left[H_{1}(\lambda), A(\mu)\right]=i \mu(A+i \mu)^{-1} i\left[H_{1}(\lambda), A\right]^{0} i \mu(A+i \mu)^{-1}
$$

$$
\begin{gathered}
s-\lim _{|\mu| \rightarrow+\infty}(-\Delta+1)^{-1 / 2} i\left[H_{1}(\lambda), A(\mu)\right](-\Delta+1)^{-1 / 2} \\
=(-\Delta+1)^{-1 / 2} i\left[H_{1}(\lambda), A\right]^{0}(-\Delta+1)^{-1 / 2}
\end{gathered}
$$

for all $\lambda \in(0,1)$, as an operator on $L^{2}$.

Using Lemma 3.2, we can also prove the following lemma in the same way as the proof of Lemma 2.5 of Weder [9].

LEMMA 3.3. Let $f \in C_{0}^{\infty}(\boldsymbol{R})$. Then

(i ) $f(H(\lambda)$ ) sends $D(A)$ into $D(A)$.

(ii) $[f(H(\lambda)), A]$ defined as operator on $D(A)$ is extended to a bounded operator on $L^{2}$ which is denoted by $[f(H(\lambda)), A]^{0}$.

LEMMA 3.4. Let $0<\lambda \ll 1$, take $f_{\lambda}(p) \in C_{0}^{\infty}(\boldsymbol{R}), 0 \leqq f_{\lambda} \leqq 1$ such that $f_{\lambda}$ has support in $(\lambda / 3,3 \lambda)$ and $f_{\lambda}=1$ on $[\lambda / 2,2 \lambda]$. Then, there exists a positive constant $C$ which is independent of $\lambda$ such that

$$
f_{\lambda}\left(H_{1}(\lambda)\right) i\left[H_{1}(\lambda), A\right]^{0} f_{\lambda}\left(H_{1}(\lambda)\right) \geqq C \lambda f_{\lambda}\left(H_{1}(\lambda)\right)^{2} .
$$

in the form sence. 
In order to show Lemma 3.4, we need the following lemma.

LEMMA 3.5. Let $u \in H^{1}\left(\boldsymbol{R}^{n}\right)$. Then, we have

$$
\left|T_{\varphi} u\right|_{L^{2}\left(R^{n-1}\right)}^{2}=\mp 2 R e \int_{\Omega_{ \pm}} u \overline{\partial_{z} u d} x .
$$

Proof. Let $u \in \boldsymbol{S}\left(\boldsymbol{R}^{n}\right)$. Noting that

$$
\int_{\Omega_{ \pm}} u \overline{\partial_{z} u} d x= \pm \int_{R^{n-1}} \int_{\varphi(y)}^{ \pm \infty} u \overline{\partial_{z} u} d z d y
$$

we have by integrating by parts

$$
\int_{\Omega_{ \pm}} u \overline{\partial_{z} u d} x= \pm \int_{R^{n-1}}\left|T_{\varphi} u\right|^{2} d y-\int_{\Omega_{ \pm}} \partial_{z} u \bar{u} d x
$$

Thus we have

$$
\int_{R^{n-1}}\left|T_{\varphi} u\right|^{2} d y=\mp 2 R e \int_{\Omega_{ \pm}} u \overline{\partial_{z} u d} x
$$

This implies lemma because $\boldsymbol{S}\left(\boldsymbol{R}^{n}\right)$ is dense in $H^{1}\left(\boldsymbol{R}^{n}\right)$.

Proof OF LEMMA 3.4. We simply write $f_{\lambda}$ instead of $f_{\lambda}\left(H_{1}(\lambda)\right)$. For $u \in$ $L^{2}$, we have (for detail, see the proof of Lemma 2.2 of Kadowaki [3])

$$
\begin{aligned}
\left\langle f_{\lambda} i\left[H_{1}(\lambda), A\right] f_{\lambda} u, u\right\rangle= & 2\left\langle\nabla f_{\lambda} u, \nabla f_{\lambda} u\right\rangle+\lambda\left\langle F_{1} f_{\lambda} u, f_{\lambda} u\right\rangle \\
& -\lambda \int_{R^{n-1}}\left(y \cdot \nabla_{y} \varphi-\varphi\right)\left(E_{1}^{+0}-E_{1}^{-0}\right)\left|T_{\varphi} f_{\lambda} u\right|^{2} d y .
\end{aligned}
$$

We estimate the second and the third term respectively. (2.3) implies that

$$
\left|\left\langle F_{1} f_{\lambda} u, f_{\lambda} u\right\rangle\right|, \quad\left|\left\langle\left(E_{1}-E_{0}\right) f_{\lambda} u, f_{\lambda} u\right\rangle\right| \leqq \delta_{0}\left|f_{\lambda} u\right|_{0}^{2},
$$

where $E_{0}(x)=a_{ \pm}^{-2}\left(x \in \Omega_{ \pm}\right)$. Let $r>0$ small enough. We decompose the third term as the following form

$$
\begin{aligned}
\int_{R^{n-1}} & \left(y \cdot \nabla_{y} \varphi-\varphi\right)\left(E_{1}^{+0}-E_{1}^{-0}\right)\left|T_{\varphi} f_{\lambda} u\right|^{2} d y \\
= & \int_{|y|<r}\left(y \cdot \nabla_{y} \varphi-\varphi\right)\left(E_{1}^{+0}-E_{1}^{-0}\right)\left|T_{\varphi} f_{\lambda} u\right|^{2} d y \\
& \quad+\int_{|y|>r}\left(y \cdot \nabla_{y} \varphi-\varphi\right)\left(E_{1}^{+0}-E_{1}^{-0}\right)\left|T_{\varphi} f_{\lambda} u\right|^{2} d y
\end{aligned}
$$

Repeating the argument in the proof of Lemma 2.1, we have

$$
\left.\left|\int_{|y|<r}(y \cdot \nabla \varphi-\varphi)\left(E_{1}^{+0}-E_{1}^{-0}\right)\right| T_{\varphi} f_{\lambda} u\right|^{2} d y \mid \leqq C r^{(n-1-\sigma p) / p}\left(\left|\nabla f_{\lambda} u\right|_{0}^{2}+\left|f_{\lambda}\right|_{0}^{2}\right),
$$

for some $p, n-1<p<(n-1) / \sigma$. 
Noting that $\chi_{|y|>r}(y)(y \cdot \nabla \varphi-\varphi)$ is bounded, by Lemma 3.5, we have

$$
\left.\left|\int_{|y|>r}(y \cdot \nabla \varphi-\varphi)\left(E_{1}^{+0}-E_{1}^{-0}\right)\right| T_{\varphi} f_{\lambda} u\right|^{2} d y \mid \leqq C\left\langle\left|f_{\lambda} u\right|,\left|\partial_{z} f_{\lambda} u\right|\right\rangle \text {. }
$$

Moreover, let $\tau>0$, small enough. Then we have

$$
\left.\left.\left|\int_{|y|>r}(y \cdot \nabla \varphi-\varphi)\left(E_{1}^{+0}-E_{1}^{-0}\right)\right| T_{\varphi} f_{\lambda} u\right|^{2} d y|\leqq \tau| f_{\lambda} u\right|_{0} ^{2}+C / \tau\left|\nabla f_{\lambda} u\right|_{0}^{2} .
$$

Using (3.3) (3.6), we can estimate the following

$$
\begin{aligned}
\langle & \left.f_{\lambda} i\left[H_{1}(\lambda), A\right]^{0} f_{\lambda} u, u\right\rangle \\
\geqq & \left(2-C \lambda r^{(n-1-\sigma p) / p}-C \lambda / \tau\right)\left|\nabla f_{\lambda} u\right|_{0}^{2}-\lambda\left(\delta_{0}+C r^{(n-1-\sigma p) / p}+\tau\right)\left|f_{\lambda} u\right|_{0}^{2} \\
= & \left(2-C \lambda r^{(n-1-\sigma p) / p}-C \lambda / \tau\right)\left\langle H_{1}(\lambda) f_{\lambda} u, f_{\lambda} u\right\rangle \\
& +\lambda\left(2-C \lambda r^{(n-1-\sigma p) / p}-C \lambda / \tau\right)\left(\left\langle\left(E_{1}-E_{0}\right) f_{\lambda} u, f_{\lambda} u\right\rangle+\left\langle\left(E_{0}-1\right) f_{\lambda} u, f_{\lambda} u\right\rangle\right) \\
& -\lambda\left(\delta_{0}+C r^{(n-1-\sigma p) / p}+\tau\right)\left|f_{\lambda} u\right|_{0}^{2} \\
\geqq & \lambda\left(2-C \lambda r^{(n-1-\sigma p) / p}-C \lambda / \tau\right) / 3\left|f_{\lambda} u\right|_{0}^{2}-\delta_{0} \lambda\left(2-C \lambda r^{(n-1-\sigma p) / p}-C \lambda / \tau\right)\left|f_{\lambda} u\right|_{0}^{2} \\
& +\lambda\left(2-C \lambda r^{(n-1-\sigma p) / p}-C \lambda / \tau\right)\left\langle\left(E_{0}-1\right) f_{\lambda} u, f_{\lambda} u\right\rangle-\lambda\left(\delta_{0}+C r^{(n-1-\sigma p) / p}+\tau\right)\left|f_{\lambda} u\right|_{0}^{2}
\end{aligned}
$$

Noting $0<\lambda, r, r, \delta_{0} \ll 1$, we have $(3.2)$.

Following Tamura [8], we consider cut off functions, $\chi_{n}(x) \in C_{0}^{\infty}\left(\boldsymbol{R}^{n}\right)$ such that $\chi_{n}(x)$ has support in $\left\{x \in \boldsymbol{R}^{n}:|x|<2\right\}$ and $\chi_{n}=1$ for $|x|<1$. For $\varepsilon>0$ small enough, we define

$$
\begin{gathered}
E_{1, \varepsilon}(x)=E_{0}(x)+\chi_{n}(\varepsilon x)\left(E_{1}(x)-E_{0}(x)\right), \\
V_{\varepsilon}^{r}(y)=\chi_{|y|>r}(y) \chi_{n-1}(\varepsilon y)\left(y \cdot \nabla_{y} \varphi(y)-\varphi(y)\right) .
\end{gathered}
$$

We further define an operator $B(\varepsilon ; \lambda)$ as

$$
\begin{aligned}
B(\varepsilon ; \lambda)= & -2 \Delta+\lambda\left(F_{1, \varepsilon}-T_{\varphi}^{*}\left(E_{1}^{+0}-E_{1}^{-0}\right) V_{\varepsilon}^{r}(y) T_{\varphi}\right. \\
& \left.-\left(R_{\varphi}^{r}\right) *\left(E_{1}^{+0}-E_{1}^{-0}\right) R_{\varphi}^{r}\right),
\end{aligned}
$$

where $F_{1, \varepsilon}=x \cdot \nabla E_{1, \varepsilon}(x)\left(x \in \Omega_{ \pm}\right), R_{\varphi}^{r}=\chi_{|y|<r}(y)\left(y \cdot \nabla_{y} \varphi(y)-\varphi(y)\right)^{1 / 2} T_{\varphi}$.

We can consider $B(\varepsilon ; \lambda)$ to be a bounded operator from $H^{1}\left(\boldsymbol{R}^{n}\right)$ to $H^{-1}\left(\boldsymbol{R}^{n}\right)$ (for detail, see the proof of Lemma 2.2 of Kadowaki [3]). For $u \in H^{2}\left(\boldsymbol{R}^{n}\right) \cap$ $D(A)$ satisfing $A u \in H^{1}\left(\boldsymbol{R}^{n}\right)$, we define the commutator $i[B(\varepsilon ; \lambda), A]$ as follows

$$
\begin{aligned}
\langle i[B(\varepsilon ; \lambda), A] u, u\rangle & =\sum_{j=1}^{3}\left\langle i\left[B_{j}(\varepsilon ; \lambda), A\right] u, u\right\rangle \\
& =\sum_{j=1}^{3}\left(\left\langle A u, B_{j}(\varepsilon ; \lambda) u\right\rangle-\left\langle B_{j}(\varepsilon ; \lambda) u, A u\right\rangle\right),
\end{aligned}
$$


where

$$
\begin{aligned}
& B_{1}(\varepsilon ; \lambda)=-2 \Delta+\lambda F_{1, \varepsilon}, \\
& B_{2}(\varepsilon ; \lambda)=-\lambda T_{\varphi}^{*}\left(E_{1}^{+0}-E_{1}^{-0}\right) V_{\varepsilon}^{r} T_{\varphi}, \\
& B_{2}(\varepsilon ; \lambda)=-\lambda\left(R_{\varphi}^{r}\right) *\left(E_{1}^{+0}-E_{1}^{-0}\right) R_{\varphi}^{r} .
\end{aligned}
$$

Following Appendix of Kadowaki [3], we show that $i[B(\varepsilon ; \lambda), A]$ is extended to a bounded oparator $H^{2}\left(\boldsymbol{R}^{n}\right)$ to $H^{-2}\left(\boldsymbol{R}^{n}\right)$. By a straightforward calculation, the form $i\left[B_{1}(\varepsilon ; \lambda), A\right]$ is extended to a bounded operator from $H^{1}\left(\boldsymbol{R}^{n}\right)$ to $H^{-1}\left(\boldsymbol{R}^{n}\right)$. Moreover we have that

$$
i\left[B_{1}(\varepsilon ; \lambda), A\right]^{0}=-4 \Delta+\lambda\left(F_{1, \varepsilon} x \cdot \nabla+\nabla^{*} \cdot x F_{1, \varepsilon}+n F_{1, \varepsilon}\right) .
$$

Lemma 2.3 implies that $i\left[B_{2}(\varepsilon ; \lambda), A\right]$ is extended to a bounded operator from $H^{1+s}\left(\boldsymbol{R}^{n}\right)$ to $H^{-1-s}\left(\boldsymbol{R}^{n}\right)$, where $s>1 / 2$. We also have

$$
\begin{aligned}
i[ & \left.B_{2}(\varepsilon ; \lambda), A\right]^{0} \\
= & -\lambda\left(T_{\varphi}^{*}\left(E_{1}^{+0}-E_{1}^{-0}\right) V_{\varepsilon}^{r} y \cdot T_{\varphi} \nabla_{y}+\left(T_{\varphi} \nabla_{y}\right) * \cdot y\left(E_{1}^{+0}-E_{1}^{-0}\right) V_{\varepsilon}^{r} T_{\varphi} \nabla_{y}\right. \\
& +T_{\varphi}^{*}\left(E_{1}^{+0}-E_{1}^{-0}\right) \varphi(y) V_{\varepsilon}^{r} T_{\varphi} \partial_{z}+\left(T_{\varphi} \partial_{z}\right) *\left(E_{1}^{+0}-E_{1}^{-0}\right) \varphi(y) V_{\varepsilon}^{r} T_{\varphi} \\
& \left.+n T_{\varphi}^{*}\left(E_{1}^{+0}-E_{1}^{-0}\right) V_{\varepsilon}^{r} T_{\varphi}\right) .
\end{aligned}
$$

We define operator $P_{\varphi}^{r}$ as

$$
P_{\varphi}^{r} u=\chi_{1 y \mid<r}(y)\left(\left(y \cdot \nabla_{y} \varphi(y)-\varphi(y)\right) \varphi(y)\right)^{1 / 2} T_{\varphi} u,
$$

for $u \in \boldsymbol{S}\left(\boldsymbol{R}^{n}\right)$. Then we can show that $P_{\varphi}^{r}$ is extended to a bounded operator from $H^{1}\left(\boldsymbol{R}^{n}\right)$ to $L^{2}\left(\boldsymbol{R}^{n-1}\right)$ (see Appendix of Kadowaki [3]). Thus we have that $i\left[B_{3}(\varepsilon ; \lambda), A\right]^{0}$ is extended to a bounded operator from $H^{2}\left(\boldsymbol{R}^{n}\right)$ to $H^{-2}\left(\boldsymbol{R}^{n}\right)$. Moreover we have

$$
\begin{aligned}
i\left[B_{3}(\varepsilon ; \lambda), A\right]^{0}= & -\lambda\left(\left(R_{\varphi}^{r}\right) *\left(E_{1}^{+0}-E_{1}^{-0}\right) y \cdot R_{\varphi}^{r} \nabla_{y}+\left(R_{\varphi}^{r} \nabla_{y}\right) * \cdot y\left(E_{1}^{+0}-E_{1}^{-0}\right) R_{\varphi}^{r}\right. \\
& +\left(P_{\varphi}^{r}\right) *\left(E_{1}^{+0}-E_{1}^{-0}\right) P_{\varphi}^{r} \partial_{z}+\left(P_{\varphi}^{r} \partial_{z}\right) *\left(E_{1}^{+0}-E_{1}^{-0}\right) P_{\varphi}^{r} \\
& \left.+n\left(P_{\varphi}^{r}\right) *\left(E_{1}^{+0}-E_{1}^{-0}\right) P_{\varphi}^{r}\right)
\end{aligned}
$$

We define an operator $i[B(\varepsilon ; \lambda), A]^{0}$ as

$$
i[B(\varepsilon ; \lambda), A]^{0}=\sum_{j=1}^{3} i\left[B_{j}(\varepsilon ; \lambda), A\right]^{0} .
$$

Thus the form $i[B(\varepsilon ; \lambda), A]^{0}$ is extended to a bounded operator from $H^{2}\left(\boldsymbol{R}^{n}\right)$ to $H^{-2}\left(\boldsymbol{R}^{n}\right)$.

LEMMA 3.6. Let $M(\varepsilon ; \lambda)=f_{\lambda}(H(\lambda)) B(\varepsilon ; \lambda) f_{\lambda}(H(\lambda))$. Then $[M(\varepsilon ; \lambda), A]$ defined as a form on $D(A)$ is extended to a bounded operator on $L^{2}$ which is 
denoted by $[M(\varepsilon ; \lambda), A]^{0}$.

Proof. The lemma is proved in exactly the same way as in the proof of Lemma 3.3 of Kadowaki [3]. We here give a brief sketch of the proof.

We write $f_{\lambda}$ for $f_{\lambda}(H(\lambda))$. Since $A(\mu)$ sends $H^{k}\left(\boldsymbol{R}^{n}\right)$ into $H^{k}\left(\boldsymbol{R}^{n}\right)(k=0$, $\pm 1, \pm 2)$, we have

$$
\begin{aligned}
& i[M(\varepsilon ; \lambda), A(\mu)] \\
& =f_{\lambda}[B(\varepsilon ; \lambda), A(\mu)] f_{\lambda}+f_{\lambda} B(\varepsilon ; \lambda)\left[f_{\lambda}, A(\mu)\right]+\left[f_{\lambda}, A(\mu)\right] B(\varepsilon ; \lambda) f_{\lambda} .
\end{aligned}
$$

Again, noting that $A(\mu)$ sends $H^{k}\left(\boldsymbol{R}^{n}\right)$ into $H^{k}\left(\boldsymbol{R}^{n}\right)(k=0, \pm 1, \pm 2)$, we have

$$
f_{\lambda}[B(\varepsilon ; \lambda), A(\mu)] f_{\lambda}=f_{\lambda} i \mu(A+i \mu)^{-1}[B(\varepsilon ; \lambda), A]^{0} i \mu(A+i \mu)^{-1} f_{\lambda}
$$

as an operator on $L^{2}$. (3.1) and (3.8) imply that

$$
s-\lim _{|\mu| \rightarrow+\infty} f_{\lambda}[B(\varepsilon ; \lambda), A(\mu)] f_{\lambda}=f_{\lambda}[B(\varepsilon ; \lambda), A]^{0} f_{\lambda}
$$

for all $\lambda \in(0,1)$, as an operator on $L^{2}$. We also prove that

$$
\begin{gathered}
s-\lim _{|\mu| \rightarrow+\infty}(-\Delta+1)^{1 / 2}\left[f_{\lambda}, A(\mu)\right](-\Delta+1)^{1 / 2} \\
=(-\Delta+1)^{1 / 2}\left[f_{\lambda}, A\right]^{0}(-\Delta+1)^{1 / 2}
\end{gathered}
$$

for all $\lambda \in(0,1)$, as an operator on $L^{2}$ (for detail, see Lemma 2.4 of Weder [9]). (3.9) and (3.10) imply that

$$
\begin{aligned}
s & =\lim _{|\mu| \rightarrow+\infty}[M(\varepsilon ; \lambda), A(\mu)] \\
& =f_{\lambda}[B(\varepsilon ; \lambda), A]^{0} f_{\lambda}+f_{\lambda} B(\varepsilon ; \lambda)\left[f_{\lambda}, A\right]^{0}+\left[f_{\lambda}, A\right]^{0} B(\varepsilon ; \lambda) f_{\lambda}
\end{aligned}
$$

for all $\lambda \in(0,1)$, as an operator on $L^{2}$. We define $[M(\varepsilon ; \lambda), A]^{0}$ by the right side of (3.11).

Finally, for $u, v \in D(A)$, we have

$$
\begin{aligned}
\left\langle[M(\varepsilon ; \lambda), A]^{0} u, v\right\rangle & =\lim _{|\mu| \rightarrow+\infty}(\langle A(\mu) u, M(\varepsilon ; \lambda) v\rangle-\langle M(\varepsilon ; \lambda) u, A(\mu) v\rangle) \\
& =\langle A u, M(\varepsilon ; \lambda) v\rangle-\langle M(\varepsilon ; \lambda) u, A v\rangle .
\end{aligned}
$$

We can prove the following lemma by a straightforward calculation.

LEMMA 3.7. As $\lambda \rightarrow 0$, one has

(i ) $\left\|(-\Delta+\lambda)^{-1 / 2}(B(\varepsilon ; \lambda)-B(\lambda))(-\Delta+\lambda)^{-1 / 2}\right\|=\varepsilon^{\theta} O(1)$,

(ii) $\left\|(-\Delta+\lambda)^{-1 / 2}((d / d \varepsilon) B(\varepsilon ; \lambda))(-\Delta+\lambda)^{-1 / 2}\right\|=\varepsilon^{\theta-1} O(1)$,

(iii) $\left\|(-\Delta+\lambda)^{-1}[B(\varepsilon ; \lambda), A]^{0}(-\Delta+\lambda)^{-1}\right\|=\varepsilon^{\theta-1} O\left(\lambda^{-1}\right)+O\left(\lambda^{-1}\right)$,

where $B(\lambda)=i\left[H_{1}(\lambda), A\right]^{0}$. 
Lemma 3.4 and 3.7 (i) imply that

$$
M(\varepsilon ; \lambda) \geqq \gamma \lambda f_{\lambda}^{2}
$$

for $\varepsilon>0$ small enough, where $\gamma>0$ is independent of $\varepsilon, \lambda$. It follows from (3.12) that $M(\varepsilon ; \lambda)$ is non-negative and hence we define an operator, $G_{\kappa}(\varepsilon ; \lambda)$, on $L^{2}$ by

$$
G_{\kappa}(\varepsilon ; \lambda)=\left(H_{1}(\lambda)-\lambda-i \kappa a^{-2}(x)-i \varepsilon M(\varepsilon ; \lambda)\right)^{-1}
$$

for $\kappa, 0<\kappa<1$ and $\varepsilon>0$ small enough.

LEMMA 3.8. There exists $\varepsilon_{0}, 0<\varepsilon_{0} \ll 1$, independent of $\lambda$ such that for $\varepsilon, 0<$ $\varepsilon \leqq \varepsilon_{0}$,

$$
\left\|G_{\kappa}(\varepsilon ; \lambda)\right\|=\varepsilon^{-1} O\left(\lambda^{-1}\right), \quad(\lambda \rightarrow 0)
$$

uniformly in $\kappa, 0<\kappa<1$.

For a proof of Lemma 3.8, see that of Lemma 5.3 of Kikuchi and Tamura [4] or Lemma 3.2 of Tamura [7].

We write

$$
F_{\kappa}(\varepsilon ; \lambda)=X_{1} G_{\kappa}(\varepsilon ; \lambda) X_{1}
$$

where $X_{1}=\left(1+|x|^{2}\right)^{-1 / 2}$.

Differentiating $F_{\kappa}(\varepsilon ; \lambda)$ in $\varepsilon$, we have

$$
(d / d \varepsilon) F_{\kappa}(\varepsilon ; \lambda)=-i X_{1} G_{\kappa} M(\varepsilon ; \lambda) G_{\kappa} X_{1}-i \varepsilon X_{1} G_{\kappa}((d / d \varepsilon) M(\varepsilon ; \lambda)) G_{\kappa} X_{1} .
$$

We can show that

$$
\begin{gathered}
G_{\kappa}(\varepsilon ; \lambda) D(A) \subset D(A) \cap H^{2}\left(\boldsymbol{R}^{n}\right), \\
\text { Range }\left(f_{\lambda} X_{1}\right) \subset D(A),
\end{gathered}
$$

(see Weder [9]).

Let $g_{\lambda}(p)=1-f_{\lambda}(p)$. We write in brief $f_{\lambda}$ and $g_{\lambda}$ for $f_{\lambda}\left(H_{1}(\lambda)\right)$ and $g_{\lambda}\left(H_{1}(\lambda)\right)$ respectively. Using (3.13) and (3.14), we can decompose $(d / d \varepsilon) F_{\kappa}(\varepsilon ; \lambda)$ as a form on $L^{2}$

$$
(d / d \varepsilon) F_{k}(\varepsilon ; \lambda)=\sum_{j=1}^{11} Y_{k}^{j}(\varepsilon ; \lambda),
$$

where

$$
\begin{aligned}
& Y_{\kappa}^{1}=-i X_{1} G_{\kappa} f_{\lambda}(B(\varepsilon ; \lambda)-B(\lambda)) f_{\lambda} G_{\kappa} X_{1}, \\
& Y_{\kappa}^{2}=i X_{1} G_{\kappa} g_{\lambda} B(\lambda) f_{\lambda} G_{\kappa} X_{1}, \\
& Y_{\kappa}^{3}=i X_{1} G_{\kappa} g_{\lambda} B(\lambda) g_{\lambda} G_{\kappa} X_{1}, \\
& Y_{\kappa}^{4}=i X_{1} G_{\kappa} f_{\lambda} B(\lambda) g_{\lambda} G_{\kappa} X_{1},
\end{aligned}
$$




$$
\begin{aligned}
& Y_{\kappa}^{5}=-i X_{1} f_{\lambda} G_{\kappa}\left[H_{1}(\lambda)-\lambda-i \kappa a(x)^{-2}-i \varepsilon M(\varepsilon ; \lambda), A\right] G_{\kappa} f_{\lambda} X_{1}, \\
& Y_{\kappa}^{6}=-i X_{1} f_{\lambda} G_{\kappa} B(\lambda) G_{\kappa} g_{\lambda} X_{1}, \\
& Y_{\kappa}^{7}=-i X_{1} g_{\lambda} G_{\kappa} B(\lambda) G_{\kappa} g_{\lambda} X_{1}, \\
& Y_{\kappa}^{8}=-i X_{1} g_{\lambda} G_{\kappa} B(\lambda) G_{\kappa} f_{\lambda} X_{1}, \\
& Y_{\kappa}^{9}=\kappa X_{1} f_{\lambda} G_{\kappa}\left[a(x)^{-2}, A\right] G_{\kappa} f_{\lambda} X_{1}, \\
& Y_{\kappa}^{10}=-i \varepsilon X_{1} G_{\kappa}((d / d \varepsilon) M(\varepsilon ; \lambda)) G_{\kappa} X_{1}, \\
& Y_{\kappa}^{11}=\varepsilon X_{1} f_{\lambda} G_{\kappa}[M(\varepsilon ; \lambda), A] G_{\kappa} f_{\lambda} X_{1} .
\end{aligned}
$$

We need the following two lemmas to estimate each term of the right side of (3.15)

LEMMA 3.9. As $\lambda \rightarrow 0$, one has:

(i) $\left\|g_{\lambda} G_{k}(\varepsilon ; \lambda)\right\|=O\left(\lambda^{-1}\right)$,

(ii) $\left\|g_{\lambda} G_{k}(\varepsilon ; \lambda)(-\Delta+\lambda)^{1 / 2}\right\|=O\left(\lambda^{-1 / 2}\right)$,

(iii) $\left\|(-\Delta+\lambda)^{1 / 2} g_{\lambda} G_{k}(\varepsilon ; \lambda)(-\Delta+\lambda)^{1 / 2}\right\|=O(1)$,

(iv) $\left\|(-\Delta+\lambda)^{1 / 2} f_{\lambda} G_{\kappa}(\varepsilon ; \lambda) X_{1}\right\|=\varepsilon^{-1 / 2}\left\|F_{\kappa}\right\|^{1 / 2} O(1)$,

(v) $\left\|(-\Delta+\lambda)^{1 / 2} g_{\lambda} G_{k}(\varepsilon ; \lambda) X_{1}\right\|=O(1)$,

(vi) $\left\|F_{\kappa}(\varepsilon ; \lambda)\right\|=\varepsilon^{-1} O(1)$,

uniformly $\kappa, 0<\kappa<1$.

For a proof of Lemma 3.9 (i ) (iii), see that of Lemma 3.5 of Tamura [7] or Lemma 5.4 of Kikuchi and Tamura [4]. Also, for a proof of (iv) and (v), see that of Lemma 3.4 of Tamura [7] or Lemma 5.5 of Kikuchi and Tamura [4]. In order to prove (vi), we need the well-known inequality

$$
\int\langle x\rangle^{-2}|u(x)|^{2} d x \leqq C \int|\nabla u(x)|^{2} d x .
$$

(3.16), (iv) and (v) imply (vi).

Noting the definition of $[M(\varepsilon ; \lambda), A]^{\circ}$ (see (3.11)) and Lemma 3.7(iii), we can prove the following lemma in the same way as the proof of Lemma 3.5 of Tamura [7] or Lemma 5.6 of Kikuchi and Tamura [4].

LEMMA 3.10. As $\lambda \rightarrow 0$, one has;

$$
\left\|[M(\varepsilon ; \lambda), A]^{0}\right\|=\varepsilon^{\theta-1} O(\lambda) .
$$

Using Lemma 3.8, 3.9 and 3.10, we can evaluate the norm of $Y_{k}^{j}, 1 \leqq j \leqq 11$, and obtain the following differential inequality (see Tamura [8]).

$$
\left\|(d / d \varepsilon) F_{\kappa}(\varepsilon ; \lambda)\right\| \leqq C\left(1+\varepsilon^{-1 / 2}\left\|F_{\kappa}\right\|^{1 / 2}+\varepsilon^{\theta-1}\left\|F_{\kappa}\right\|\right) .
$$


Let $\varepsilon_{0}, 0<\varepsilon_{0} \ll 1$. Then by Lemma 3.9 (vi), we have

$$
\left\|F_{\kappa}\left(\varepsilon_{0} ; \lambda\right)\right\|=\varepsilon_{0}^{-1} O(1), \quad(\lambda \rightarrow 0) .
$$

(3.17) and (3.18) imply

$$
\left\|F_{\kappa}(0 ; \lambda)\right\|=O(1), \quad(\lambda \rightarrow 0),
$$

uniformly $\kappa, 0<\kappa<1$. The proof of Lemma 2.2 is now complete.

\section{References}

[1] D. Eidus, The principle of limiting amplitude, Russ. Math. Surv 24 (1969), 97-167.

[2] - The limiting absorption and amplitude principle for the diffraction problem with two unbounded media, Comm. Math. Phys 107 (1986), 29-38.

[3] M. Kadowaki, The limiting absorption principle for the acoustic wave operators in two unbounded media, Tsukuba J. Math. 17 (1993), 345-362.

[4] K. Kikuchi and H. Tamura, Limiting amplitude principle for acoustic propagators in perturbed stratified fluids, J. Defferential Equations 93 (1991), 260-282.

[5] E. Mourre, Absence of singular continuous spectrum for certain self-adjoint operators, Comm. Math. Phys 78 (1981), 391-408.

[6] P. Perry, I. M. Sigal and B. Simon, Spectral analysis of $N$-body Schrödinger operators, Ann. of Math 114 (1981), 519-567.

[7] H. Tamura, Resolvent estimates at low frequencies and limiting amplitude princile for acoustic propagator, J. Math. Soc. Japan 41 (1989), 549-575.

[8] - - Principle of limiting absorption for $N$-body Schrödinger operators, A remark on the commutator method, Lett. in Math Phys 17 (1989), 31-36.

[9] R. Weder, Spectral analysis of strongly propagator systems, J. Reine Angew. Math. 354 (1984), 95-122.

University of Tsukuba,

Institude of Mathematics

Ibaraki, 305 Japan

Current adress : $1-5-3$, Tsurumaki,

Tama, Tokyo, 206,

Japan 